Antti H. Niemi. 2009. A bilinear shell element based on a refined shallow shell model. Espoo, Finland. 30 pages. Helsinki University of Technology Institute of Mathematics Research Reports, A562. International Journal for Numerical Methods in Engineering, submitted for publication.

(C) 2009 by author and (C) 2009 John Wiley \& Sons

Preprinted with permission. 
Helsinki University of Technology Institute of Mathematics Research Reports

\title{
A BILINEAR SHELL ELEMENT BASED ON A REFINED SHALLOW SHELL MODEL
}

\author{
Antti H. Niemi
}



Helsinki University of Technology Institute of Mathematics Research Reports

\section{A BILINEAR SHELL ELEMENT BASED ON A REFINED SHALLOW SHELL MODEL}

Antti H. Niemi 
Antti H. Niemi: A bilinear shell element based on a refined shallow shell model; Helsinki University of Technology Institute of Mathematics Research Reports A562 (2009).

\begin{abstract}
A four-node shell finite element of arbitrary quadrilateral shape is developed and applied to the solution of static and vibration problems. The element incorporates five generalized degrees of freedom per node, namely the three displacements of the curved middle surface and the two rotations of its normal vector. The stiffness properties of the element are defined using isoparametric principles in a local coordinate system with axes approximately parallel to the edges of the element. A distinct feature of the present formulation is the derivation of the geometric curvatures from the interpolated normal vector so as to enable explicit coupling between bending and stretching in the strain energy functional. In addition, the bending behavior of the element is improved with numerical modifications which include mixed interpolation of the membrane and transverse shear strains. The numerical experiments show that the element is able to compete in accuracy with the highly reputable bilinear elements of the commercial codes ABAQUS and ADINA.
\end{abstract}

AMS subject classifications: $74 \mathrm{~S} 05,74 \mathrm{~K} 25$

Keywords: shells, finite elements, locking

\title{
Correspondence
}

Antti H. Niemi

Helsinki University of Technology

Department of Mathematics and Systems Analysis

P.O. Box 1100

FI-02015 TKK

Finland

antti.h.niemi@tkk.fi

ISBN 978-951-22-9719-1 (print)

ISBN 978-951-22-9720-7 (PDF)

ISSN 0784-3143 (print)

ISSN 1797-5867 (PDF)

Helsinki University of Technology

Faculty of Information and Natural Sciences

Department of Mathematics and Systems Analysis

P.O. Box 1100, FI-02015 TKK, Finland

email: math@tkk.fi http://math.tkk.fi/ 


\section{Introduction}

In this paper, we introduce a four node shell element that has its roots in classical shell theory but is defined using standard isoparametric techniques and geometric initial data. In particular, no explicit reference to mathematical objects such as charts or Christoffel symbols is made in the formulation. Numerical models of this kind have actually been proposed in the early finite element literature concerning shells but those elements were usually cast in the framework of Kirchhoff's hypothesis and neglected transverse shear deformation. For a discussion of such formulations, see e.g. [1, 2] and the references therein.

Today, the majority of finite elements that are employed in engineering applications are shear deformable elements of the Reissner-Mindlin type which are based on the so-called degenerated solid approach pioneered by Ahmad et. al in [3]. At the beginning, the simplest and the most intriguing element of this quadrilateral family, namely the element with four nodes, was omitted from the published literature - probably because of its poor accuracy. Later on, the bilinear element has anyway become the front runner thanks to the various extensions of the degeneration procedure proposed by different authors. Seminal research in this respect was done by MacNeal [4] and Dvorkin \& Bathe [5]. It seems that the transverse shear interpolation proposed by Dvorkin \& Bathe is nowadays a standard practice in four node shell elements. The basic formulation, referred to as MITC4, is a central part of the finite element code ADINA and forms the backbone of the bilinear shell elements of ABAQUS as well.

The motivation of the present work comes mainly from a recent study [6] which predicts the rising of parametric error amplification or locking phenomenon when approximating boundary or interior layer effects in shell deformations using bilinear elements like MITC4. Here we demonstrate that this is indeed the case by utilizing ABAQUS and ADINA in benchmark computations involving real non-shallow shells.

According to the mentioned prior study, locking at layers is a consequence of the crude representation of shell kinematics using bilinear degenerated elements and hence difficult to avoid completely without taking the geometric curvature into account within each element. Our present experiments strengthen this conclusion. Namely, the membrane behavior of MITC4 and its relatives can be improved by different methods, see e.g. [7, 8, 9], but usually the strains are still computed from the bilinear geometry representation so that the manner in which they are defined in the first place is not altered. The formulation proposed here supplements the methodology in this respect by employing strain fields that are straightforward approximations to those of Reissner-Naghdi shell theory. Especially the effect of geometric curvature on strains is computed explicitly by using the interpolated normal vector. Actually, a similar approach was taken by Gebhardt and Scheizerhof in [10], but their method suffers from severe membrane locking and has not gained much attention. In our formulation the strain energy of each element is in- 
stead computed by applying locally a refined shallow shell model which was derived by Malinen in [11]. The corresponding membrane and transverse shear strains are then interpolated very carefully in order to suppress the locking effects.

\section{$2 \quad$ Numerical shell model}

\subsection{Geometry}

The first step in the derivation of the geometrically compatible formulation is to rectify the straight-sided quadrilateral element in cases where its four corner nodes $\mathbf{r}_{1}, \mathbf{r}_{2}, \mathbf{r}_{3}, \mathbf{r}_{4}$ are not coplanar. This can be accomplished by projecting the element to the plane which passes through the element midpoint $\mathbf{r}_{c}=\frac{1}{4}\left(\mathbf{r}_{1}+\mathbf{r}_{2}+\mathbf{r}_{3}+\mathbf{r}_{4}\right)$ and is parallel to the diagonals $\mathbf{d}_{1}=\mathbf{r}_{3}-\mathbf{r}_{1}$ and $\mathbf{d}_{2}=\mathbf{r}_{2}-\mathbf{r}_{4}$ joining the corner nodes. Following MacNeal in [4], we define the directions of the local co-ordinates $x, y$ by

$$
\mathbf{e}_{x}=\frac{\widehat{\mathbf{d}}_{1}+\widehat{\mathbf{d}}_{2}}{\left|\widehat{\mathbf{d}}_{1}+\widehat{\mathbf{d}}_{2}\right|}, \quad \mathbf{e}_{y}=\frac{\widehat{\mathbf{d}}_{1}-\widehat{\mathbf{d}}_{2}}{\left|\widehat{\mathbf{d}}_{1}-\widehat{\mathbf{d}}_{2}\right|}
$$

where $\widehat{\mathbf{d}}_{1}=\mathbf{d}_{1} /\left|\mathbf{d}_{1}\right|$ and $\widehat{\mathbf{d}}_{2}=\mathbf{d}_{2} /\left|\mathbf{d}_{2}\right|$. With this choice the local coordinate system can only rotate by multiples of 90 degrees when the node sequence changes.

The local co-ordinates $\left(x_{i}, y_{i}\right)$ of node $i$ are given by

$$
x_{i}=\left(\mathbf{r}_{i}-\mathbf{r}_{c}\right) \cdot \mathbf{e}_{x}, \quad y_{i}=\left(\mathbf{r}_{i}-\mathbf{r}_{c}\right) \cdot \mathbf{e}_{y}
$$

and the planar element so obtained is denoted by $K$. We assume that all interior angles of $K$ are less than 180 degrees so that the reference element $\hat{K}=(-1,1) \times(-1,1)$ can be mapped onto $K$ by one-to-one transformation

$$
x(\xi, \eta)=\sum_{i=1}^{4} x_{i} N_{i}(\xi, \eta), \quad y(\xi, \eta)=\sum_{i=1}^{4} y_{i} N_{i}(\xi, \eta)
$$

where $N_{i}$ are the standard bilinear shape functions defined on $\hat{K}$ :

$$
\begin{array}{ll}
N_{1}(\xi, \eta)=\frac{1}{4}(1-\xi)(1-\eta), & N_{2}(\xi, \eta)=\frac{1}{4}(1+\xi)(1-\eta) \\
N_{3}(\xi, \eta)=\frac{1}{4}(1+\xi)(1+\eta), & N_{4}(\xi, \eta)=\frac{1}{4}(1-\xi)(1+\eta)
\end{array}
$$

The derivatives of the shape functions with respect to the co-ordinates $x, y$ are computed using the Jacobian matrix $\mathbf{J}$ as follows

$$
\left[\begin{array}{l}
\frac{\partial N_{i}}{\partial x} \\
\frac{\partial N_{i}}{\partial y}
\end{array}\right]=\mathbf{J}^{-T}\left[\begin{array}{c}
\frac{\partial N_{i}}{\partial \xi} \\
\frac{\partial N_{i}}{\partial \eta}
\end{array}\right], \quad \mathbf{J}=\left[\begin{array}{ll}
\frac{\partial x}{\partial \xi} & \frac{\partial x}{\partial \eta} \\
\frac{\partial y}{\partial \xi} & \frac{\partial y}{\partial \eta}
\end{array}\right]
$$




\subsection{Kinematics}

The stiffness properties of the element are formulated in terms of five independent degrees of freedom per node. These consist of the three displacements $U_{i}, V_{i}, W_{i}$ of the middle surface and the two rotations $\Theta_{i}, \Psi_{i}$ of its normal vector $\mathbf{e}_{3}^{(i)}$. This unit vector is specified as initial data (usually referred to as the nodal director) and the two orthogonal directions $\mathbf{e}_{1}^{(i)}$ and $\mathbf{e}_{2}^{(i)}$ tangent to the middle surface can then be generated in different ways, see e.g. $[12,13,14,9]$.

In our case the actual displacement interpolation within each element is introduced separately for the tangential and normal components of the middle surface displacements. For this purpose we will assume that the global degrees of freedom $\left(U_{i}, V_{i}, W_{i}\right)$ are already related to the triad $\left(\mathbf{e}_{1}^{(i)}, \mathbf{e}_{2}^{(i)}, \mathbf{e}_{3}^{(i)}\right)$. The transformation of the local degrees of freedom $u_{i}, v_{i}, w_{i}$ to the global ones is then performed such that

$$
u_{i} \mathbf{e}_{x}+v_{i} \mathbf{e}_{y}=U_{i} \mathbf{e}_{1}^{(i)}+V_{i} \mathbf{e}_{2}^{(i)}, \quad w_{i}=W_{i}
$$

The local rotational degrees of freedom $\theta_{i}, \psi_{i}$ are defined in the same way as $u_{i}, v_{i}$ so that the whole displacement transformation may be written as

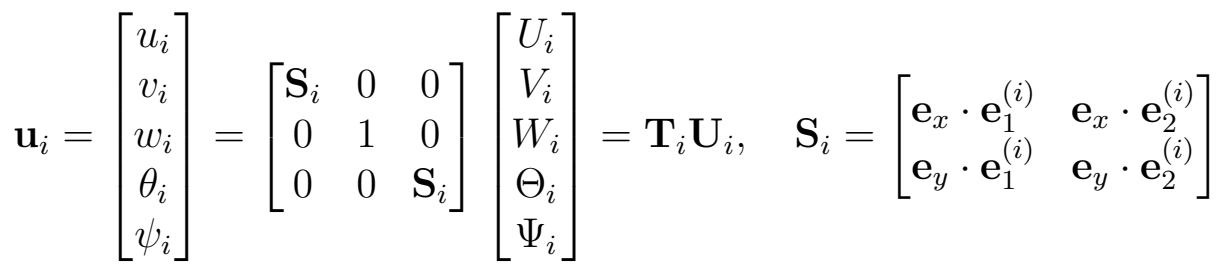

These displacement components are then interpolated using standard bilinear shape functions:

$$
\mathbf{u}(\xi, \eta)=\left[\begin{array}{c}
u(\xi, \eta) \\
v(\xi, \eta) \\
w(\xi, \eta) \\
\theta(\xi, \eta) \\
\psi(\xi, \eta)
\end{array}\right]=\sum_{i=1}^{4} \mathbf{u}_{i} N_{i}(\xi, \eta)
$$

In case of homogeneous and isotropic material with Young's modulus $E$ and Poisson ratio $\nu$, the deformation energy of the shell over $K$ may be written (approximately, see the remarks below) as

$$
\begin{aligned}
\frac{1}{2} \mathcal{A}_{K}(\mathbf{u}, \mathbf{u}) & =\frac{E t}{2\left(1-\nu^{2}\right)} \int_{K}\left[\nu\left(\beta_{11}+\beta_{22}\right)^{2}+(1-\nu)\left(\beta_{11}^{2}+2 \beta_{12}^{2}+\beta_{22}^{2}\right)\right] \mathrm{d} x \mathrm{~d} y \\
& +k \frac{E t}{4(1+\nu)} \int_{K}\left[\gamma_{1}^{2}+\gamma_{2}^{2}\right] \mathrm{d} x \mathrm{~d} y \\
& +\frac{E t^{3}}{24\left(1-\nu^{2}\right)} \int_{K}\left[\nu\left(\kappa_{11}+\kappa_{22}\right)^{2}+(1-\nu)\left(\kappa_{11}^{2}+2 \kappa_{12}^{2}+\kappa_{22}^{2}\right)\right] \mathrm{d} x \mathrm{~d} y
\end{aligned}
$$


Here $t$ is the (constant) thickness of the shell and $k$ is an additional (optional) shear correction factor. The quantities $\beta_{i j}, \gamma_{i}$ and $\kappa_{i j}$ denote the components of the membrane strain, transverse shear strain and bending strain tensors, respectively. These are given by

$$
\begin{aligned}
& \beta_{11}=\frac{\partial u}{\partial x}+b_{11} w \\
& \beta_{22}=\frac{\partial v}{\partial y}+b_{22} w \\
& \beta_{12}=\frac{1}{2}\left(\frac{\partial u}{\partial y}+\frac{\partial v}{\partial x}\right)+b_{12} w
\end{aligned}
$$

and

$$
\begin{aligned}
& \gamma_{1}=\theta-\frac{\partial w}{\partial x}+b_{11} u+b_{12} v \\
& \gamma_{2}=\psi-\frac{\partial w}{\partial y}+b_{12} u+b_{22} v
\end{aligned}
$$

and

$$
\begin{aligned}
\kappa_{11} & =\frac{\partial \theta}{\partial x}-b_{12}\left(\frac{\partial v}{\partial x}+b_{12} w\right) \\
\kappa_{22} & =\frac{\partial \psi}{\partial y}-b_{12}\left(\frac{\partial u}{\partial y}+b_{12} w\right) \\
\kappa_{12} & =\frac{1}{2}\left[\frac{\partial \theta}{\partial y}+\frac{\partial \psi}{\partial x}-b_{11}\left(\frac{\partial u}{\partial y}+b_{12} w\right)-b_{22}\left(\frac{\partial v}{\partial x}+b_{12} w\right)\right]
\end{aligned}
$$

Here $b_{i j}$ denote the components of the curvature tensor of the shell over $K$. These can be approximated using the interpolated normal vector

$$
\mathbf{n}(\xi, \eta)=\sum_{i=1}^{4} \mathbf{e}_{3}^{(i)} N_{i}(\xi, \eta)
$$

as

$$
b_{11}=\mathbf{e}_{x} \cdot \frac{\partial \mathbf{n}}{\partial x}, \quad b_{22}=\mathbf{e}_{y} \cdot \frac{\partial \mathbf{n}}{\partial y}, \quad b_{12}=\mathbf{e}_{x} \cdot \frac{\partial \mathbf{n}}{\partial y}
$$

Remark 1. The model (4)-(7) may be viewed as a local approximation of the classical geometrically compatible Reissner-Naghdi model, see $[11,15]$. The relative error of the deformation energy is expected to be of order $\mathcal{O}\left(h_{K} / R\right)$, where $h_{K}$ is the diameter of $K$ and $R$ denotes the minimum principal radius of curvature of the shell middle surface over $K$. Moreover, the relative truncation error arising from the computation of $b_{i j}$ using (8) and (9) is generally only of order $\mathcal{O}\left(h_{K} / R\right)$ and not better. Anyway, these errors (in the initial data) are expected to be insignificant compared to the numerical discretization error of $\mathbf{u}$ using bilinear shape functions.

Remark 2. The bending strains (7) are not tensorially invariant under general transformations of the co-ordinates $x, y$. However, it is easy to check 
that $\kappa_{i j}$ transform correctly if the local coordinate system is only rotated by multiple of 90 degrees. Consequently, the resulting stiffness matrix will not depend on node sequencing when the local basis vectors are defined by (1). If desired, the tensorial invariance could be retained under more general co-ordinate transformations by adding certain linear combinations of the membrane strains $\beta_{i j}$ to the expressions of the bending strains, cf. [11]. Anyway, it seems that this type of orthodoxy is not required because the added terms would have a negligible effect on the strain energy when $t$ is small.

\subsection{Numerical strain reductions}

The standard bilinear scheme based on (3)-(9) suffers from parametric locking effects because of its inability to reproduce inextensional deformation states with vanishing membrane and transverse shear strains. As a remedy, we modify the critical terms $\beta_{i j}$ and $\gamma_{i}$ in the strain energy functional numerically as $\beta_{i j} \hookrightarrow \beta_{i j, h}$ and $\gamma_{i} \hookrightarrow \gamma_{i, h}$.

The "shear trick" $\gamma_{i} \hookrightarrow \gamma_{i, h}$ consists of two steps. In the first step the transverse shear strain vector $\gamma$ is projected to the space

$$
\mathbf{S}(K)=\left\{\boldsymbol{\eta}=\mathbf{J}^{-T} \widehat{\boldsymbol{\eta}}, \quad \widehat{\boldsymbol{\eta}} \in \mathbf{S}(\hat{K})\right\}
$$

where $\mathbf{S}(\hat{K})$ is spanned by $\left[\begin{array}{ll}1 & 0\end{array}\right]^{T},\left[\begin{array}{ll}0 & 1\end{array}\right]^{T},\left[\begin{array}{ll}\eta & 0\end{array}\right]^{T}$ and $\left[\begin{array}{ll}0 & \xi\end{array}\right]^{T}$. The interpolation projection operator into $\mathbf{S}(K)$ is denoted by $\boldsymbol{\Lambda}_{K}$ and is defined by the conditions

$$
\left(\boldsymbol{\Lambda}_{K} \boldsymbol{\rho}-\boldsymbol{\rho}\right) \cdot \mathbf{t}=0 \text { at the midpoint of every edge } E \text { of } K
$$

Here $\mathbf{t}$ is the tangent vector to the edge $E$.

In the second step the (dimensionless) shear correction factor is redefined as

$$
k \hookrightarrow k_{K}=\frac{t^{2}}{t^{2}+\alpha_{K} h_{K}^{2}}
$$

where $h_{K}$ is a characteristic dimension of $K$ and $\alpha_{K} \geq 0$ is a stabilization parameter to be chosen. We take $h_{K}$ to be the length of the shortest edge of $K$ and set $\alpha_{K}=0.5\left(1-\tau_{K}\right)$, where $\tau_{K}$ is the so called taper ratio of $K$, $0<\tau_{K} \leq 1$. It measures the deviation of $K$ from a rectangle and is defined as

$$
\tau_{K}=\frac{4 \min _{i} \mu\left(T_{i}\right)}{\sum_{i=1}^{4} \mu\left(T_{i}\right)}
$$

where $\mu\left(T_{i}\right), i=1,2,3,4$ denote the areas of the four triangles which are formed by subdividing $K$ with its diagonals, see Figure 1 . Consequently, we will have $\alpha_{K}=0$, i.e. no stabilization, for rectangular elements with $\tau_{K}=1$ and $\alpha_{K} \approx 0.1$ for relatively distorted elements with $\tau_{K} \approx 0.8$.

The "stretching trick" $\beta_{i j} \hookrightarrow \beta_{i j, h}$ is performed in a similar way. The membrane strain tensor $\boldsymbol{\beta}$ is projected into the space $\mathbf{M}(K)$ spanned by

$$
\left\{\left[\begin{array}{ll}
1 & 0 \\
0 & 0
\end{array}\right],\left[\begin{array}{ll}
0 & 0 \\
0 & 1
\end{array}\right],\left[\begin{array}{cc}
0 & 1 \\
1 & 0
\end{array}\right],\left[\begin{array}{cc}
\psi_{1} & \frac{1}{2} \psi_{2} \\
\frac{1}{2} \psi_{2} & 0
\end{array}\right],\left[\begin{array}{cc}
0 & \frac{1}{2} \psi_{1} \\
\frac{1}{2} \psi_{1} & \psi_{2}
\end{array}\right]\right\}
$$




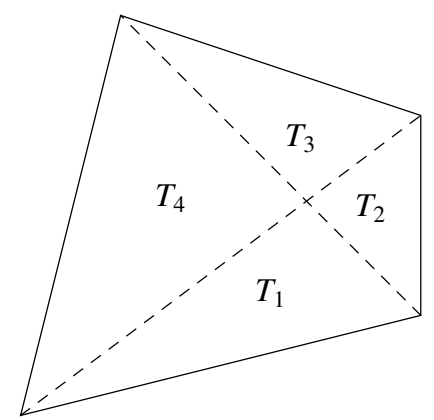

Figure 1: Subdivision of $K$ used in the definition of the taper ratio $\tau_{K}$.

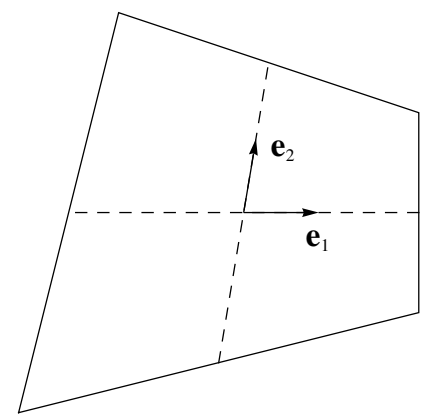

Figure 2: The basis vectors $\mathbf{e}_{1}$ and $\mathbf{e}_{2}$ used in the definition of $\boldsymbol{\Pi}_{K}$.

using a projector operator $\boldsymbol{\Pi}_{K}$ which is defined by the interpolation conditions

$$
\begin{aligned}
\mathbf{t} \cdot\left(\boldsymbol{\Pi}_{K} \boldsymbol{\beta}-\boldsymbol{\beta}\right) \mathbf{t}=0 & \text { at the midpoint of every edge } E \text { of } K \\
\mathbf{e}_{1} \cdot\left(\boldsymbol{\Pi}_{K} \boldsymbol{\beta}-\boldsymbol{\beta}\right) \mathbf{e}_{2}=0 & \text { at the midpoint of } K
\end{aligned}
$$

Here $\mathbf{e}_{1}$ and $\mathbf{e}_{2}$ are tangent vectors to the lines joining the midpoints of opposite edges of $K$, see Figure 2. The strains $\psi_{1}$ and $\psi_{2}$ in (11) are defined by

$$
\psi_{1}=\frac{\partial \Xi}{\partial x}, \quad \psi_{2}=\frac{\partial \Xi}{\partial y}
$$

where $\Xi$ is the "hourglass" mode which has the values $\Xi_{i}=\{+1,-1,+1,-1\}$ at the nodes, cf. $[16,17]$.

Furthermore, the reduced membrane shear strain $\beta_{12, h}$ is evaluated at the center of the element $(\xi=\eta=0)$ and this value is used in the evaluation of the strain energy functional at the $2 \times 2$ set of Gauss points. The necessity of this additional modification follows from the analysis of [6].

Remark 3. In case of a flat plate, the above shear strain reduction and stabilization correspond to the so called stabilized MITC4 element introduced by Lyly, Stenberg and Vihinen in [18]. When $\alpha_{K}$ is set to zero (like for rectangular elements), the MITC4 element of Bathe and Dvorkin is obtained. 
Remark 4. In case of a curved shell, the above treatment of transverse shear strains differs slightly from the one used in the original MITC4 shell element even if $\alpha_{K}=0$. Here the interpolation is based on the tangential components of the $2 \mathrm{D}$ transverse shear strains (6) whereas the original formulation relies on the isoparametric geometry approximation and interpolates the covariant transverse shear components of the corresponding Green-Lagrange strain tensor. According to [11], the outcome of both procedures is virtually the same at least for nearly rectangular elements.

Remark 5. For smooth deformations and nearly rectangular elements the above "stretching trick" leads to membrane strains that are also very close to those arising from the bilinear geometry representation applied in MITC4, see [11]. On the other hand, notable differences between the formulations are expected in cases where anisotropically varying displacement modes, such as boundary layers or vibration modes, are being captured by using elongated elements, see [6].

\subsection{Linear static analysis}

The element stiffness matrix $\mathbf{K}_{\mathbf{u}}$ corresponding to the local degrees of freedom $\mathbf{u}_{i}$ is obtained through

$$
\mathcal{A}_{K}(\mathbf{u}, \mathbf{u})=\mathbf{z}^{T} \mathbf{K}_{\mathbf{u}} \mathbf{z}, \quad \mathbf{z}=\left[\begin{array}{llll}
\mathbf{u}_{1} & \mathbf{u}_{2} & \mathbf{u}_{3} & \mathbf{u}_{4}
\end{array}\right]^{T}
$$

and its contribution is assembled to the global stiffness matrix $\mathbf{K}$ using the co-ordinate transformation (2) as

$$
\mathbf{K} \leftarrow \mathbf{T}^{T} \mathbf{K}_{\mathbf{u}} \mathbf{T}, \quad \mathbf{T}=\left[\begin{array}{llll}
\mathbf{T}_{1} & & & \\
& \mathbf{T}_{2} & & \\
& & \mathbf{T}_{3} & \\
& & & \mathbf{T}_{4}
\end{array}\right]
$$

The finite element equations of static equilibrium are

$$
\mathbf{K Z}=\mathbf{F}
$$

where $\mathbf{Z}=\left[\mathbf{U}_{1} \ldots \mathbf{U}_{N}\right]^{T}$ is the vector of unknown displacements at each unconstrained node of the finite element mesh and the vector $\mathbf{F}$ represents external loading.

For instance, a distributed surface traction can be represented by concentrated forces $\mathbf{f}_{i}=\left(f_{U}^{(i)}, f_{V}^{(i)}, f_{W}^{(i)}\right)$ acting at the nodes. The element load vector $\mathbf{F}_{\mathbf{U}}$ is then calculated using the potential energy

$$
\mathcal{L}_{K}(\mathbf{U})=\int_{K} \mathbf{f} \cdot \mathbf{U} \mathrm{d} x \mathrm{~d} y=\mathbf{z}^{T} \mathbf{F}_{\mathbf{U}}
$$

and bilinear interpolation for $\mathbf{f}$. The contribution can be assembled to the consistent load vector directly as

$$
\mathbf{F} \leftarrow \mathbf{F}_{\mathbf{u}}
$$




\subsection{Frequency analysis}

The natural frequencies and vibration modes of the shell are computed from the generalized eigenvalue problem

$$
\mathbf{K} \boldsymbol{\Phi}_{i}=\omega_{i}^{2} \mathbf{M} \boldsymbol{\Phi}_{i}
$$

where $\mathbf{M}$ is the mass matrix and $\omega_{i}$ and $\boldsymbol{\Phi}_{i}$ are the angular frequency and mode shape, respectively, for mode $i$.

The element mass matrix $\mathbf{M}_{\mathbf{u}}$ is defined as

$$
\begin{aligned}
\mathcal{M}_{K}(\mathbf{u}, \mathbf{u}) & =\rho t \int_{K}\left(u^{2}+v^{2}+w^{2}\right) \mathrm{d} x \mathrm{~d} y+\rho \frac{t^{3}}{12} \int_{K}\left(\theta^{2}+\psi^{2}\right) \mathrm{d} x \mathrm{~d} y \\
& =\mathbf{z}^{T} \mathbf{M}_{\mathbf{u}} \mathbf{z}
\end{aligned}
$$

and its contribution to the global mass matrix is given by

$$
\mathbf{M} \leftarrow \mathbf{T}^{T} \mathbf{M}_{\mathbf{u}} \mathbf{T}
$$

analogously to (12). Here we have assumed for simplicity that the mass density $\rho$ is constant.

\section{Benchmark computations}

The primary objective of this section is to demonstrate the usage of the bilinear shell element presented above in some selected test problems. The secondary objective is to compare the accuracy of the new formulation with the established four node elements of the finite element codes ABAQUS and ADINA. Especially the asymptotic behavior of the different formulations is investigated when the shell thickness approaches the limit value of zero.

Our first test concerns the classical pinched cylinder with rigid diaphragm support at the two ends. This is a challenging problem where the shell carries the external loading by a rather complex combination of membrane and bending actions. On the other hand, the diaphragm support resembles closely periodic boundary conditions at the two ends so that some analytical insight of the deformation can be obtained using Fourier analysis, see Appendix.

In the second test a shell structure of the shape of a hyperbolic paraboloid is pinched in turn. The edges are assumed to be completely fixed here and we shall rely on numerical reference solutions (Appendix). In principle, the resulting load-carrying mechanism is similar to that of the pinched cylinder but the deformation now contains some special features characteristic to hyperbolic shells.

The dynamic capabilities of the formulations are tested in the last two problems by means of eigenvalue analysis. These problems involve the same structures as above so that analytical solution is available for the cylinder and numerical reference solution can be computed for the hyperbolic shell.

The various bilinear shell finite element formulations that were evaluated are listed below: 
MITC4. This archetypical four-noded isoparametric shell element was introduced by Dvorkin and Bathe in [5]. The formulation utilizes mixed interpolation of the covariant transverse shear strain components whereas the remaining in-layer strains are computed using the standard displacement interpolations. The element is available in the ADINA System whose version 8.5 was used in our comparison tests [19].

MITC4IM. The membrane properties of MITC4 can be improved by supplementing the associated in-plane displacements with the so called incompatible modes, see [9]. In fact, this enhancement is used by default in ADINA System starting from version 8.4.

S4. This is a general-purpose bilinear element that is included in the shell element library of ABAQUS/Standard, see [20]. The formulation assumes a similar transverse shear strain field as MITC4 but employs one point integration plus hourglass stabilization in the evaluation of the transverse shear energy. The membrane strains of S4 are fully integrated but treated with an assumed strain method which is closely related to the concept of incompatible displacement modes.

S4R. This is a one point quadrature element with specialized membrane and bending hourglass control. The handling of transverse shear strains is identical with S4. It would seem that S4R is currently more or less the default shell element of ABAQUS.

MITC4S (Present). The formulation introduced in Section 2 is given here the label MITC4S to maintain some consistence with the naming conventions used in earlier works of the author and collaborators, see $[21,16,6]$. An academic implementation of the element was performed using Wolfram Mathematica 6.

\subsection{Static problems}

\section{Pinched cylinder with end diaphragms}

Consider a circular cylinder of radius $R=100$ and length $2 L=200$, the shell middle surface being described by the equation

$$
Y^{2}+Z^{2}=R^{2}, \quad-L<X<L
$$

in the global Cartesian $X Y Z$-coordinate system. The Poisson ratio and Young modulus are set to $\nu=0.3$ and $E=3 \cdot 10^{7}$. The cylinder is loaded by two normal point loads of magnitude $F=1$ located centrally at the opposite sides of the structure, see Figure 3.

Exploiting symmetry only one eighth of the cylinder was numerically analyzed. The corresponding boundary conditions can be imposed here in 


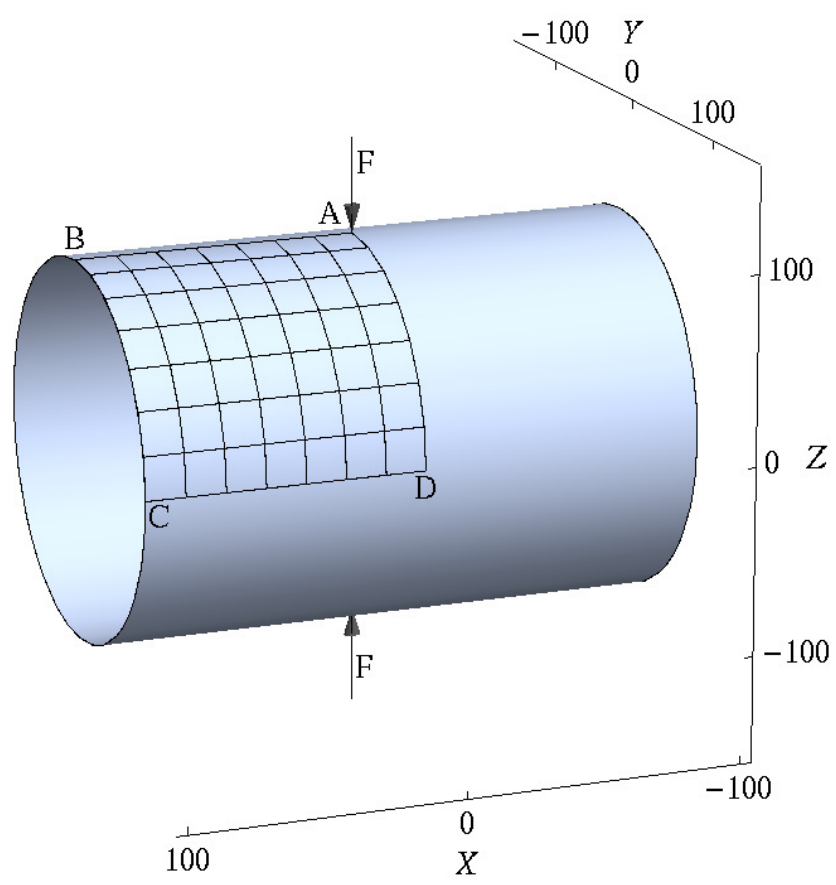

Figure 3: Pinched cylinder.

terms of the generalized displacement components in the global Cartesian coordinate directions as follows:

$$
\begin{aligned}
& U_{Y}=\Phi_{X}=\Phi_{Z}=0 \text { on } A B \\
& U_{Y}=U_{Z}=\Phi_{X}=0 \text { on } B C \\
& U_{Z}=\Phi_{X}=\Phi_{Y}=0 \text { on } C D \\
& U_{X}=\Phi_{Y}=\Phi_{Z}=0 \text { on } A D
\end{aligned}
$$

Moreover, only one fourth of the total load magnitude is directed at the computational domain $A B C D$.

Firstly, the numerical calculations were carried out for a shell with $R / t=$ 100 using $7 \times 7,14 \times 14$ and $28 \times 28$ grids of elements. In addition to canonical uniform subdivisions, two families of non-uniform grids were used as well in the computations. First of these was formed by refining the mesh towards the load application point $A$ so that the element width along each edge increases geometrically away from $A$. In the second version the refinement was performed merely along the edges adjacent to $A$ while keeping the subdivisions of $B C$ and $C D$ as uniform. The value $r=10$ was used for the ratio of the largest and the smallest element width along the refined edges. The coarsest mesh of each sequence is shown in Figure 4.

The numerical results displaying the convergence of the displacement under the point load, which is linearly related to the strain energy, are reported in Figures 5-7. The finite reference value used here was obtained from a double Fourier series solution of the classical shell equations, see Appendix 

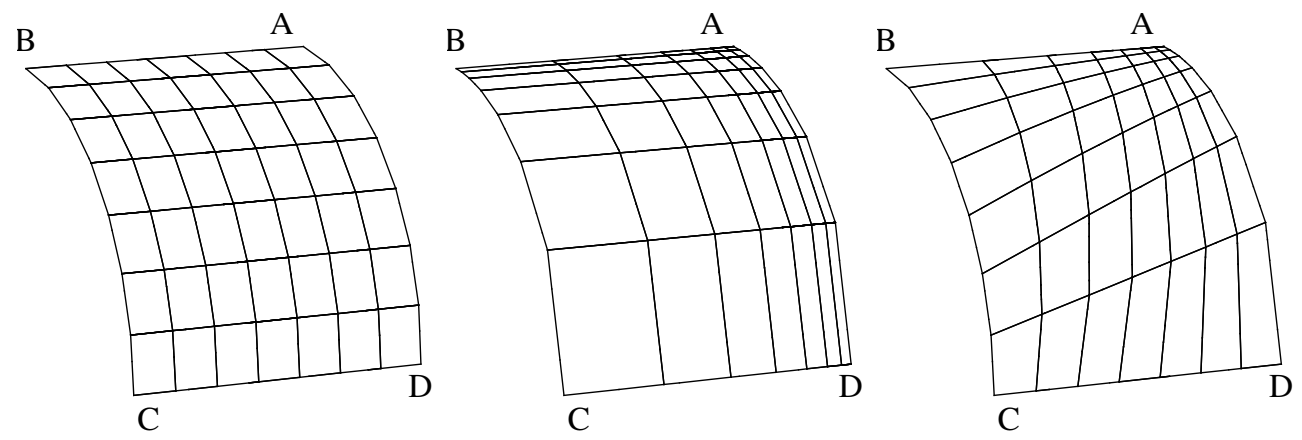

Figure 4: Discretizations of the pinched cylinder using uniform, uniformly refined and non-uniformly refined meshes.

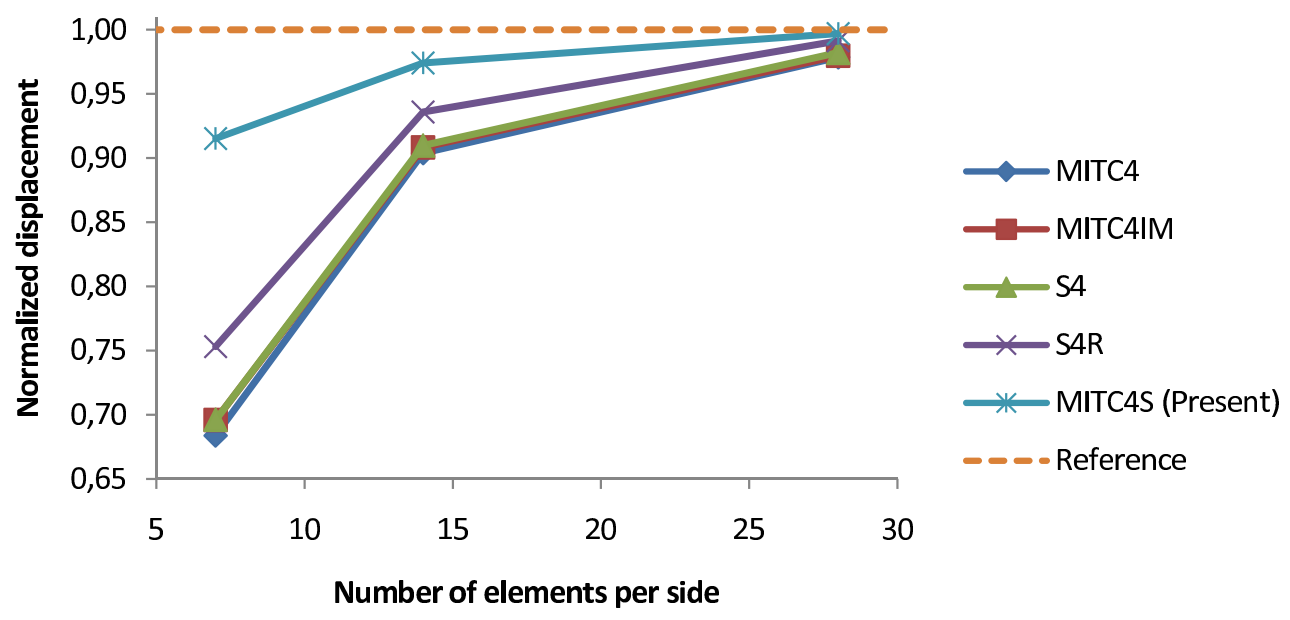

Figure 5: Strain energy convergence of the pinched cylindrical shell: uniform meshes at $R / t=100$.

and also $[1,22]$. These results indicate that the present formulation is superior to the geometrically incompatible formulations in terms of coarse-mesh accuracy. Nevertheless, each element reaches the reference value up to the accuracy of $2 \%$ as the mesh spacing diminishes - S4R slightly faster than the other four-node elements of ABAQUS and ADINA especially on rectangular grids.

The asymptotic behavior of the different formulations was investigated by decreasing gradually the value of the thickness parameter $t$. The computations were performed using refined $28 \times 28$ meshes which were concentrated further towards the load application point $A$ by setting $r=33,100$ and 333 corresponding to the radius to thickness ratio $R / t=1000,10000$ and 100000 . We point out that the most dominant part of the deformation is expected to be restricted into the range of $\sim \sqrt{R t}$ from $A$, see Figure 8 and [21, 23].

The normalized displacement values under the point load are shown in 


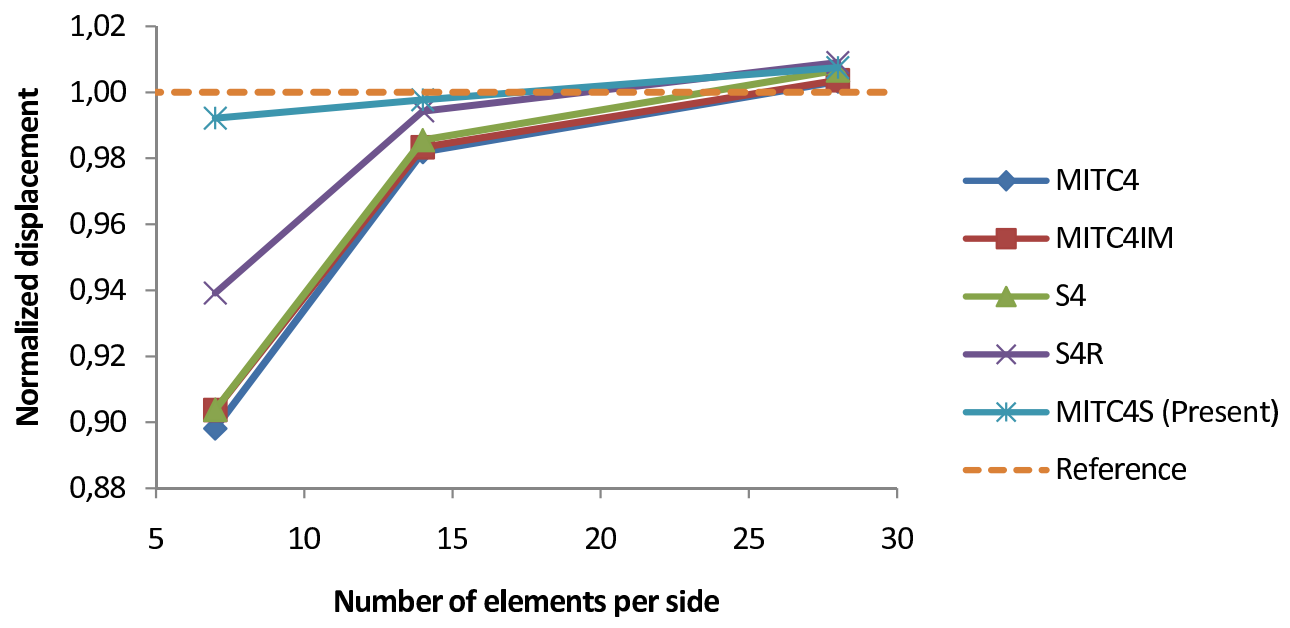

Figure 6: Strain energy convergence of the pinched cylindrical shell: uniformly refined meshes at $R / t=100$.

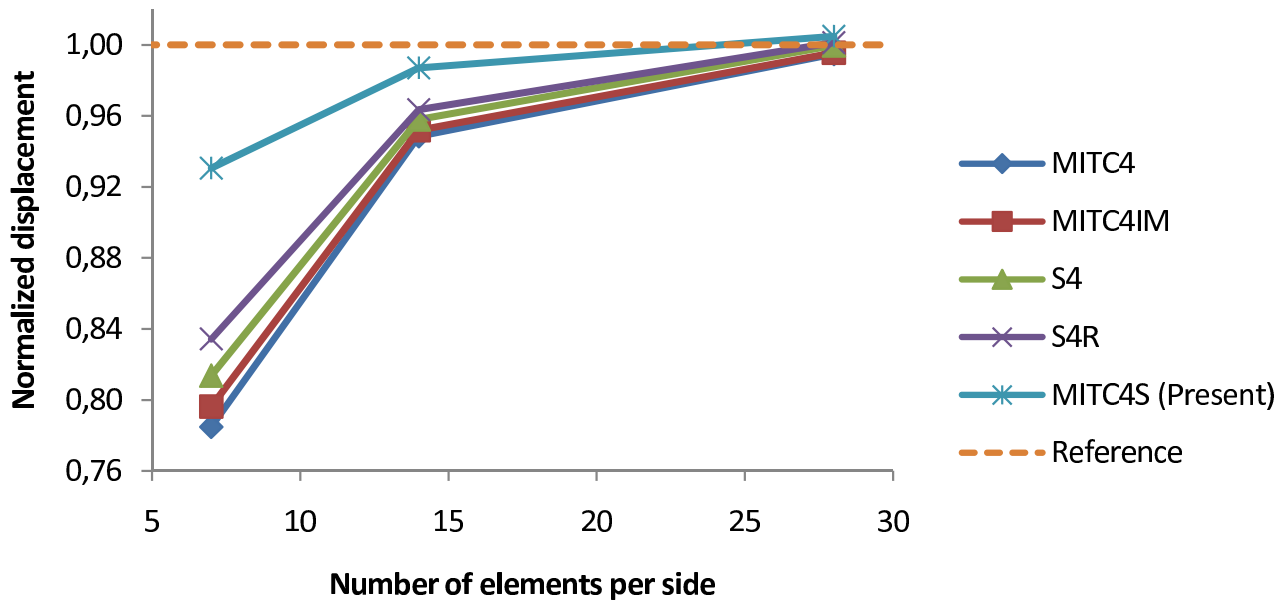

Figure 7: Strain energy convergence of the pinched cylindrical shell: nonuniformly refined meshes at $R / t=100$. 

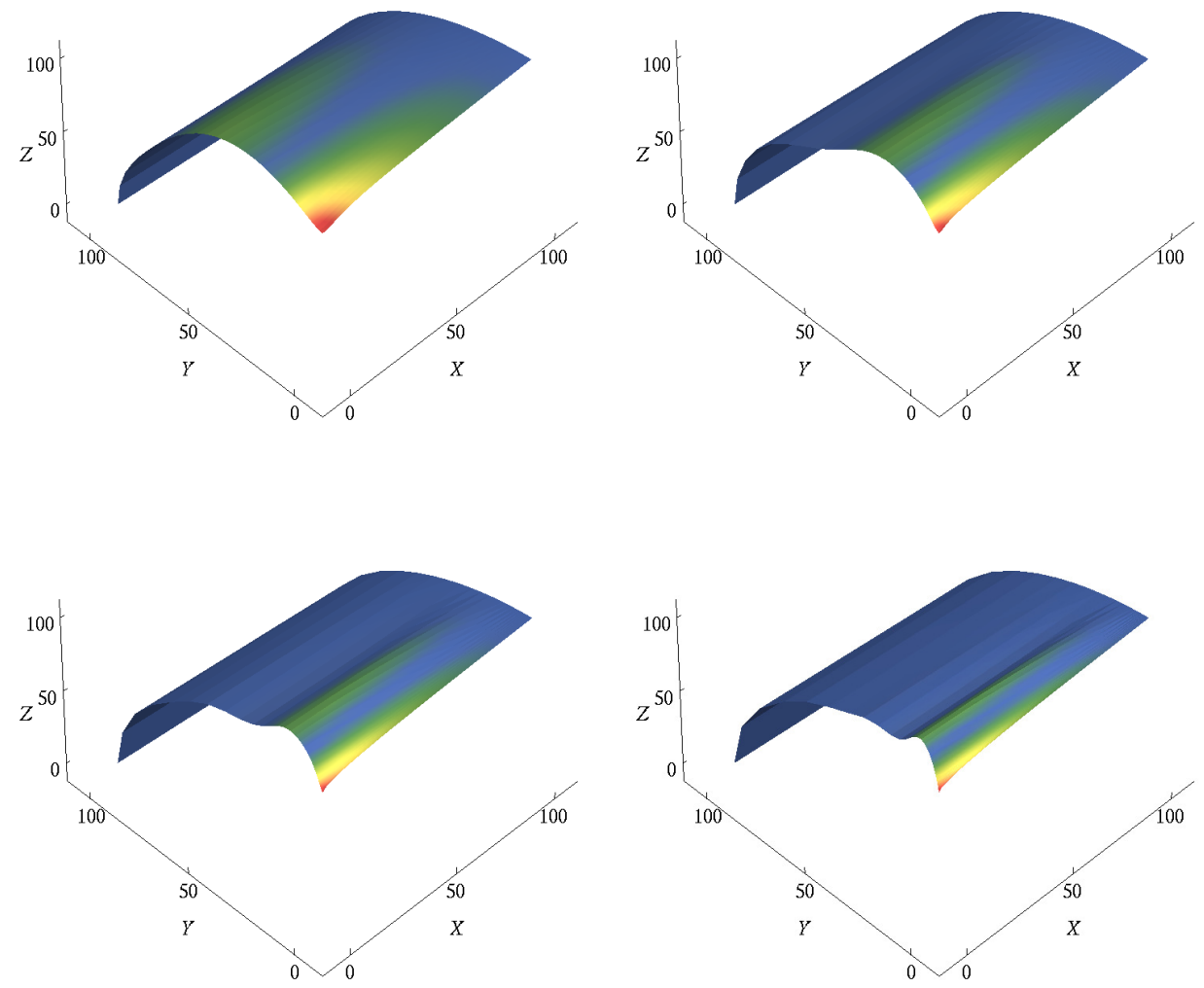

Figure 8: Deformation of the pinched cylindrical shell: uniformly refined $28 \times 28$ meshes of MITC4S elements at $R / t=100,1000,10000$ and 100000 . 


\begin{tabular}{llllll}
\hline$R / t$ & MITC4 & MITC4IM & S4 & S4R & MITC4S (Present) \\
\hline 1000 & 0.99 & 0.99 & 0.99 & 0.99 & 1.00 \\
10000 & 0.98 & 0.98 & 0.98 & 0.99 & 0.99 \\
100000 & 0.95 & 0.98 & 0.98 & 0.99 & 0.99 \\
\hline
\end{tabular}

Table 1: Normalized displacement of the pinched cylindrical shell: uniformly refined meshes with 28 elements per side.

\begin{tabular}{llllll}
\hline$R / t$ & MITC4 & MITC4IM & S4 & S4R & MITC4S (Present) \\
\hline 1000 & 0.95 & 0.95 & 0.96 & 0.97 & 0.99 \\
10000 & 0.81 & 0.82 & 0.86 & 0.92 & 1.00 \\
100000 & 0.54 & 0.55 & 0.60 & 0.79 & 0.94 \\
\hline
\end{tabular}

Table 2: Normalized displacement of the pinched cylindrical shell: nonuniformly refined meshes with 28 elements per side.

Tables 1 and 2 for the uniformly and non-uniformly refined meshes. On the uniformly refined mesh, all elements except for MITC4 preserve their accuracy also when the thickness is reduced. The locking of MITC4 is probably a consequence of the inability of bilinear degenerated elements to capture the long-range angular layer modes of cylindrical shells, see [6]. In this case such a layer develops near the generator of the cylinder passing through the load application point as shown in Figure 8. Apparently the method of incompatible displacement modes used in MITC4IM and S4 as well as the one-point quadrature of S4R are able to correct this defect.

On the non-uniformly refined meshes, all four-node elements of ABAQUS and ADINA lose the relative accuracy of $2 \%$ already at $R / t=1000$ and finish with unbearable error levels of $21-46 \%$ at $R / t=100000$. On the other hand, the accuracy of MITC4S is satisfactory almost uniformly with respect to $R / t$ also here.

\section{Pinched hyperbolic paraboloid with clamped edges}

In this test we consider a hyperbolic paraboloid described by the equation

$$
Z=\frac{1}{R} X Y, \quad-L<X, Y<L
$$

in the Cartesian coordinate system. Here $R=100$ is the radius of twist and $L=100$ stands for the length of the structure whose material parameters are taken from the previous problem. The structure is loaded by a single normal point load at $(X, Y, Z)=(0,0,0)$ while restraining the edges completely from moving.

Only one quarter of the shell (Figure 9) was numerically modeled using the symmetry conditions

$$
\begin{aligned}
& U_{X}=\Phi_{X}=0 \text { on } A B \\
& U_{Y}=\Phi_{Y}=0 \text { on } A D
\end{aligned}
$$




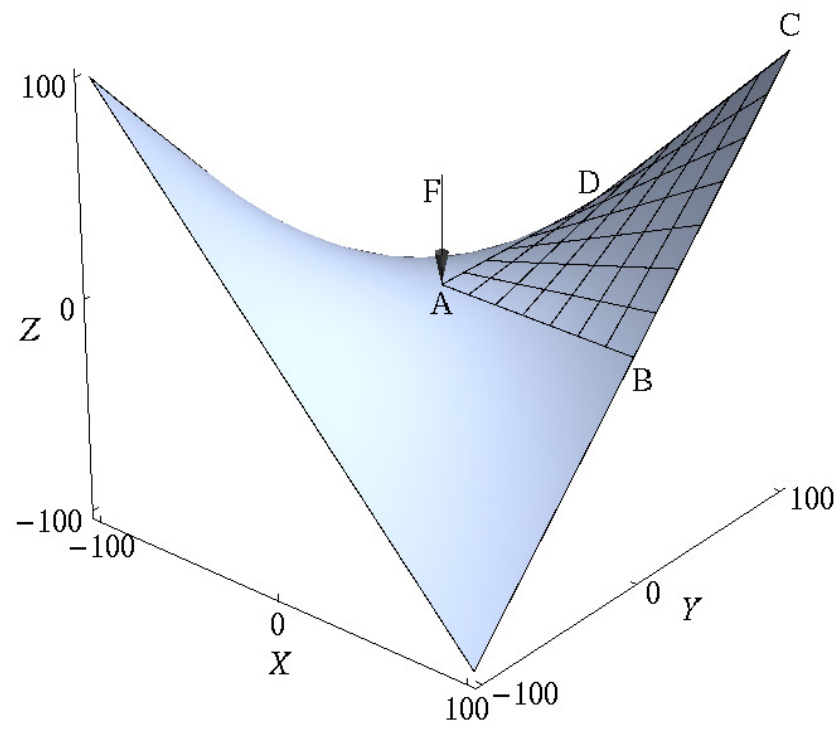

Figure 9: Pinched hyperbolic paraboloid.

We start again by setting $t=1$ and by examining the strain energy convergence of the formulations on mesh sequences analogous to the ones used for the pinched cylinder - see Figure 10 for the $7 \times 7$ mesh of each type. The results of Figures 11-13 are quite similar to those obtained in the previous benchmark problem, the advantage of the geometrically compatible approach being obvious especially on the coarsest grid. In this case there is no clear order of superiority among the other competitors.

We move now on to the asymptotic convergence tests by reducing the thickness and re-refining the meshes precisely in the same way as described earlier. The normalized displacement values for the two types of refined meshes are reported in Tables 3 and 4 . This time the accuracy of all geomet-
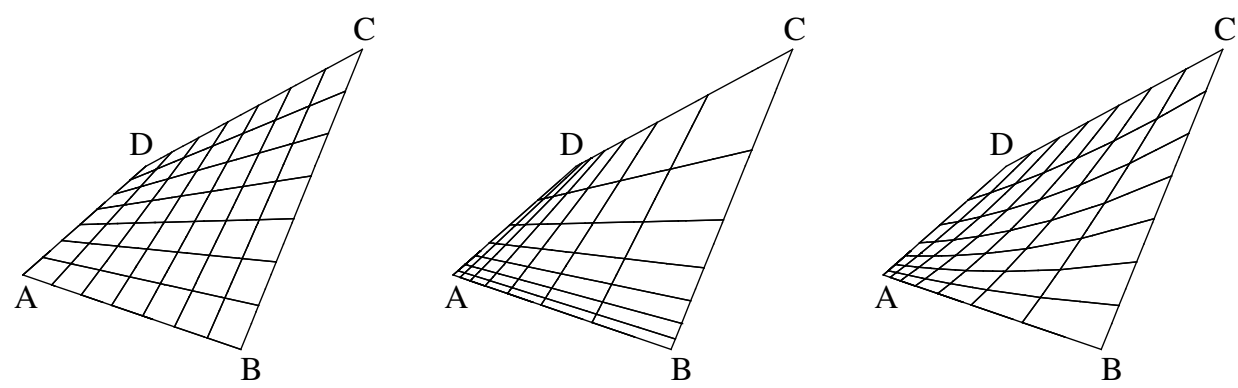

Figure 10: Discretizations of the pinched hyperbolic paraboloid using uniform, refined rectangular and refined non-rectangular meshes. 


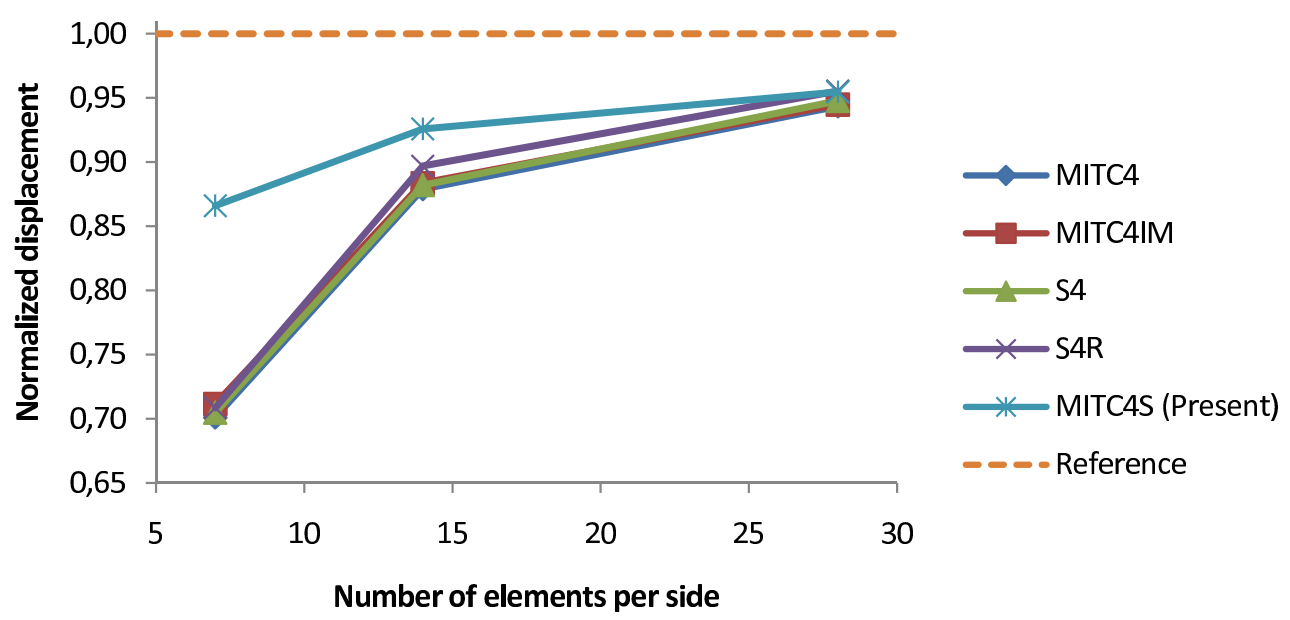

Figure 11: Strain energy convergence of the pinched hyperbolic paraboloid: uniform meshes at $R / t=100$.

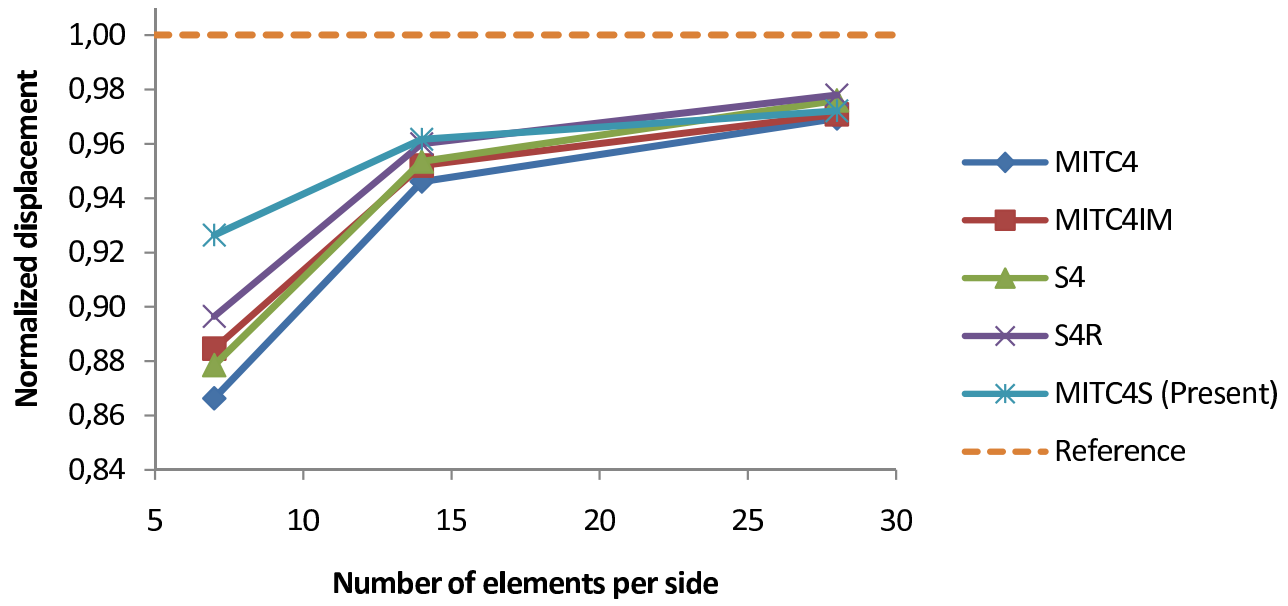

Figure 12: Strain energy convergence of the pinched hyperbolic paraboloid: uniformly refined meshes at $R / t=100$. 


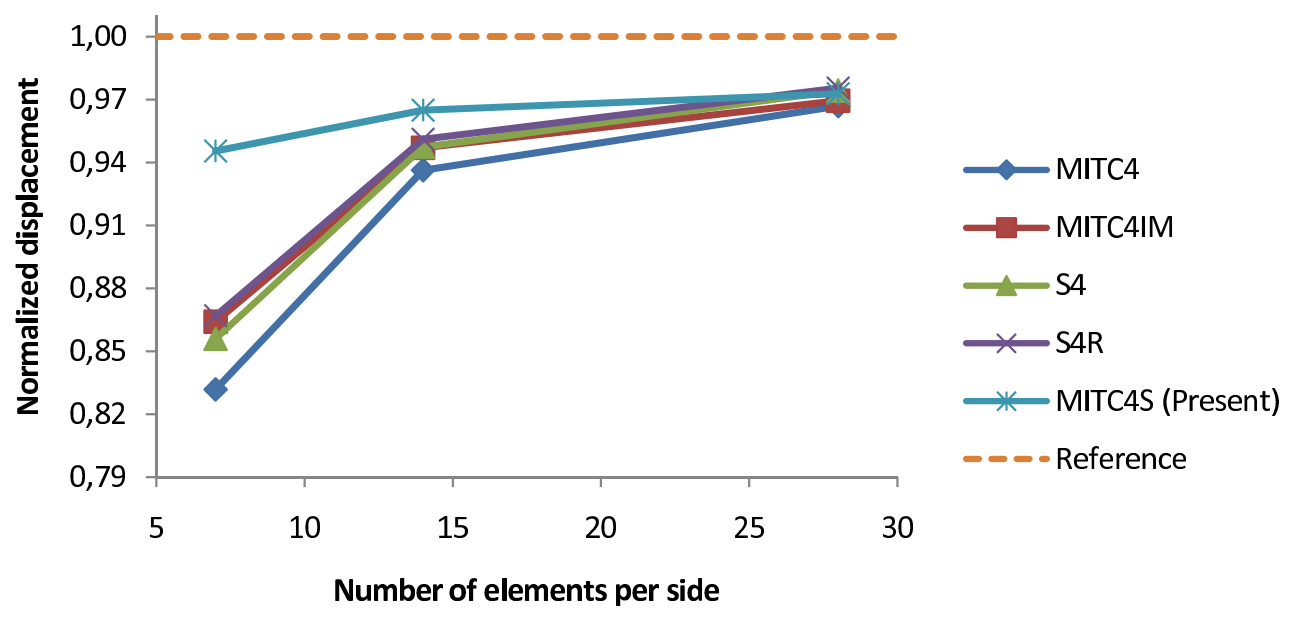

Figure 13: Strain energy convergence of the pinched hyperbolic paraboloid: non-uniformly refined meshes at $R / t=100$.

\begin{tabular}{llllll}
\hline$R / t$ & MITC4 & MITC4IM & S4 & S4R & MITC4S (Present) \\
\hline 1000 & 0.98 & 0.99 & 0.98 & 0.99 & 0.99 \\
10000 & 0.93 & 0.97 & 0.94 & 0.98 & 0.99 \\
100000 & 0.82 & 0.92 & 0.83 & 0.97 & 0.99 \\
\hline
\end{tabular}

Table 3: Normalized strain energy of the pinched hyperbolic paraboloid: uniformly refined meshes with 28 elements per side.

rically incompatible elements deteriorates already on the uniformly refined meshes. The phenomenon is supposedly related to the approximation of the characteristic layers that develop near the generators of the hyperbolic surface passing through the load point, see Figure 14.

As a matter of fact, a rather severe error amplification has been theoretically predicted in [6] concerning the approximation of line layers in hyperbolic shells using bilinear degenerated elements. It would seem that even S4R cannot wriggle out of this situation completely despite of its one-point quadrature. In any case the present formulation with reduced geometrically compatible strains appears uniformly convergent as anticipated in [6].

On the non-uniformly refined meshes, the accuracy of the bilinear elements in ABAQUS and ADINA deteriorates from the error level of 2-3\% at $R / t=1000$ to $7-25 \%$ at $R / t=100000$. The relative error of MITC4S stays under $2 \%$ uniformly with respect to the thickness at the same time.

\subsection{Dynamical problems}

\section{Cylinder with end diaphragms}

Consider once more the cylindrical shell described above and constrained kinematically as in (13). In this test, we utilize as a benchmark the mini- 


\begin{tabular}{llllll}
\hline$R / t$ & MITC4 & MITC4IM & S4 & S4R & MITC4S (Present) \\
\hline 1000 & 0.97 & 0.98 & 0.98 & 0.98 & 1.00 \\
10000 & 0.90 & 0.96 & 0.92 & 0.97 & 0.99 \\
100000 & 0.75 & 0.85 & 0.79 & 0.93 & 0.98 \\
\hline
\end{tabular}

Table 4: Normalized strain energy of the pinched hyperbolic paraboloid: non-uniformly refined meshes with 28 elements per side.
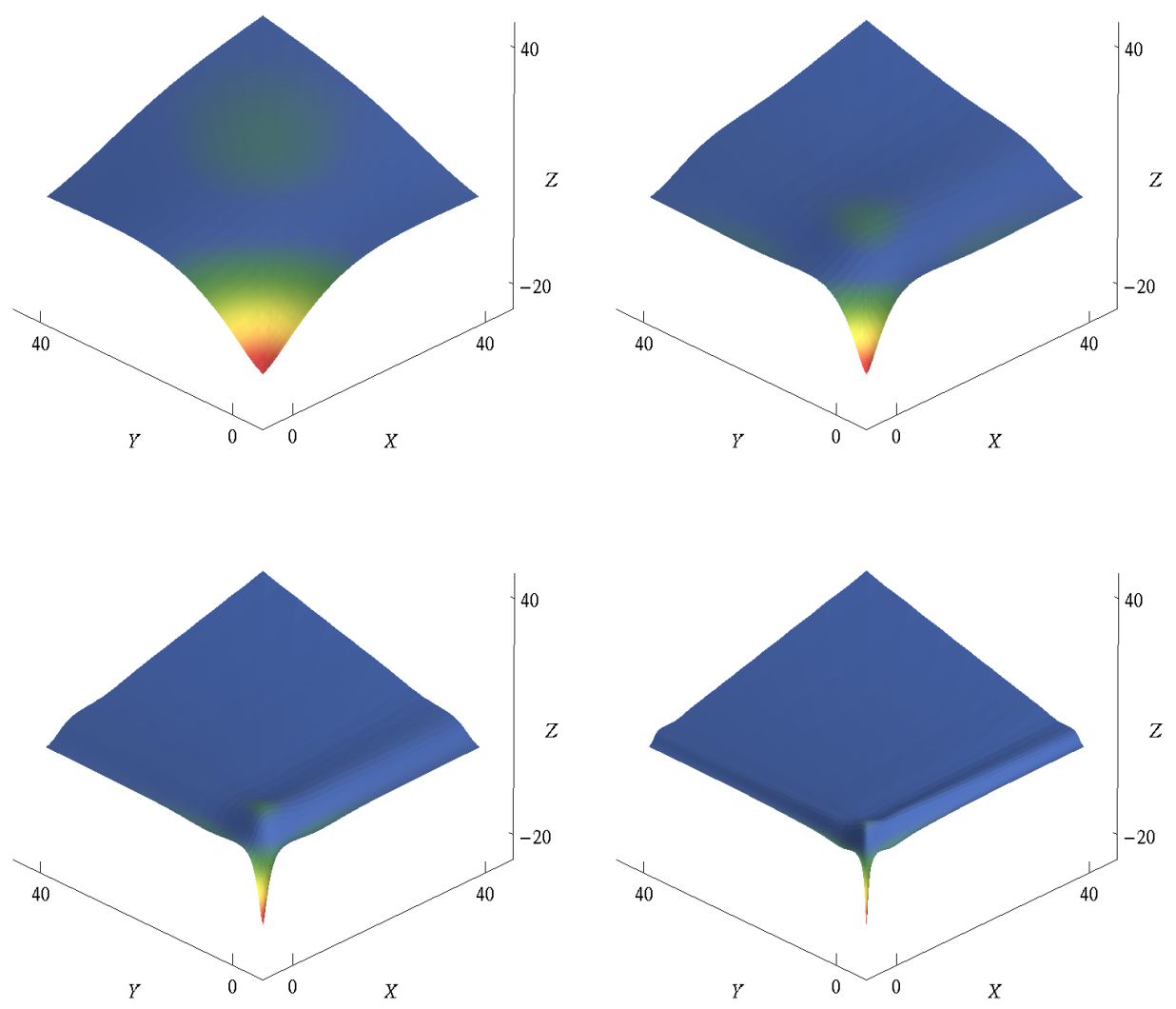

Figure 14: Deformation of the pinched hyperbolic paraboloid: uniformly refined $28 \times 28$ meshes of MITC4S elements at $R / t=100,1000,10000$ and 100000 (only part of the computational domain is shown). 


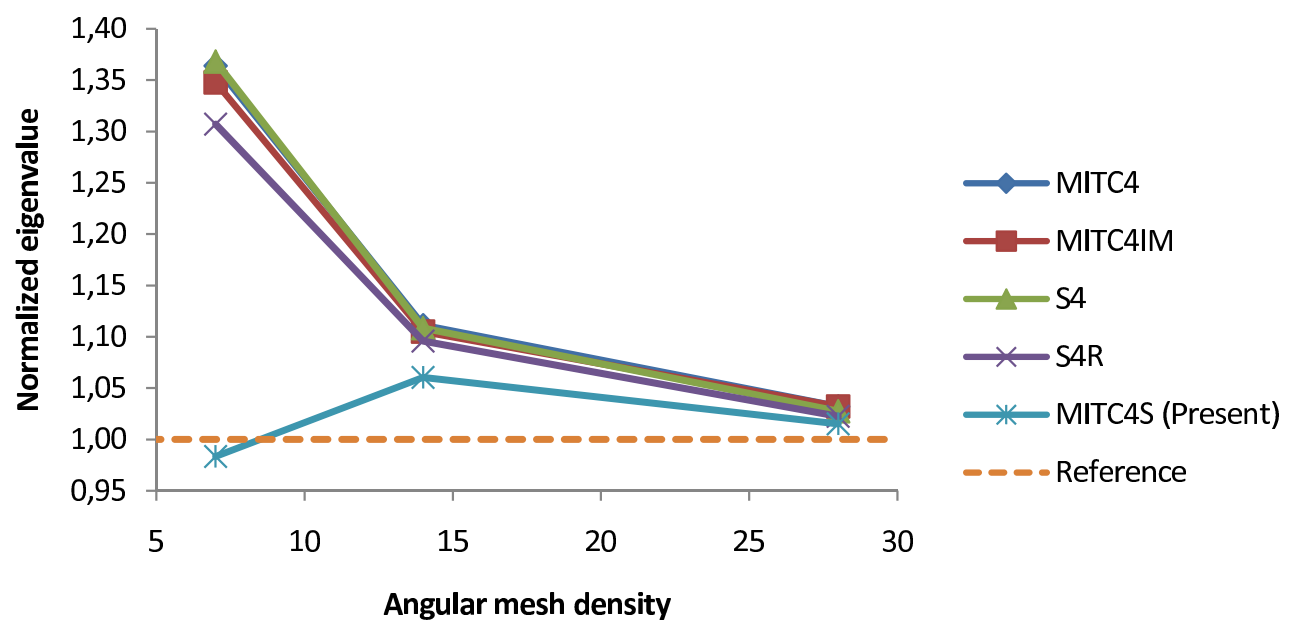

Figure 15: Convergence of the lowest eigenvalue of the cylindrical shell at $R / t=100$ : uniform $7 \times N$ meshes with $N=7,14,28$.

mum eigenvalue $\lambda=\min _{i} \omega_{i}^{2}$ corresponding to the fundamental frequency of natural vibrations of the structure, see Section 2.5. The mass density of the shell is taken to be $\rho=0.3$ and different values of the thickness were again used in the computations.

Let us begin with the most realistic situation where $R / t=100$. A sequence of uniform $7 \times N$ meshes with $N=7,14,28$ was used here to check the convergence behavior of the lowest eigenvalue with respect to the angular mesh spacing. The results are shown in Figure 15. On a coarse mesh, the advantage of MITC4S over the non-curved ones is very clear. But note that its convergence is not monotonic in this problem setup.

Concerning the asymptotic behavior of the eigenproblem as $t / R$ becomes smaller, the results in [24] show in particular that (see also Appendix)

1. The axial profile of the lowest eigenmode is virtually independent of $t$

2. The lowest eigenmode oscillates in the angular direction with a wave length proportional to $\left(L^{2} R t\right)^{1 / 4}$

Here these oscillations are followed up by employing uniform $7 \times N$ meshes where the angular mesh density $N$ attains the values 32, 58 and 104 when $R / t$ equals 1000, 10000 and 100000, respectively, see Figure 16.

The corresponding eigenvalues, which have been computed using the different formulations and normalized with respect to the analytical values obtained from Fourier analysis (see Appendix), are reported in Table 5. Firstly, the performance of the elements seems to be independent of the thickness excluding MITC4 which overestimates the lowest eigenvalue and hence the fundamental frequency rather severely when the thickness is small. Obviously here the locking effect arises from the oscillation of the vibration mode in the angular direction in the same way as in the approximation of the 

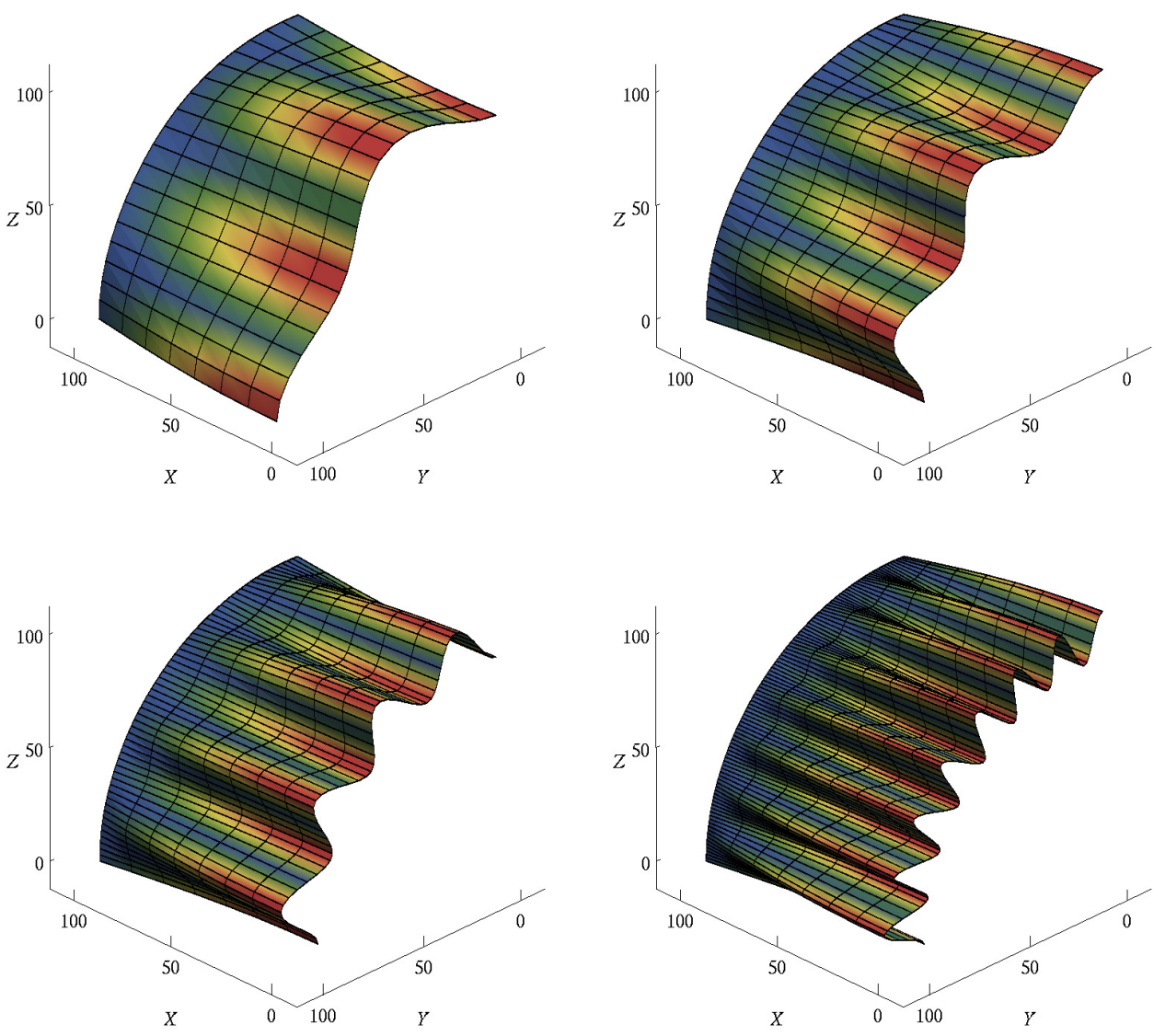

Figure 16: Lowest eigenmode of the cylindrical shell at $R / t=100,1000$, 10000 and 100000: uniform $7 \times N$ meshes of MITC4S elements with $N=19$, 32,58 and 104 . 


\begin{tabular}{llllll}
\hline$R / t$ & MITC4 & MITC4IM & S4 & S4R & MITC4S (Present) \\
\hline 1000 & 1.08 & 1.06 & 1.06 & 1.05 & 1.01 \\
10000 & 1.15 & 1.05 & 1.05 & 1.04 & 0.99 \\
100000 & 1.36 & 1.05 & 1.05 & 1.04 & 1.00 \\
\hline
\end{tabular}

Table 5: Lowest eigenvalues of the cylindrical shell (normalized to unity).

angular layer modes, see $[24,6]$. Secondly, the enhanced geometrically incompatible elements also lag behind MITC4S in terms of their prediction accuracy. Indeed, for the lowest eigenvalue a relative accuracy of $1 \%$ can barely be reached using a $14 \times 58$ mesh of S4R elements when $R / t=1000$ so that almost quadruple number of degrees of freedom are required to overtake MITC4S in this case.

\section{Hyperbolic paraboloid with clamped edges}

As a final numerical test, we compute the lowest eigenmodes of the hyperbolic paraboloid structure using the same material parameters, computational domain and kinematic constraints as before. At the beginning, the eigenmodes corresponding to the different thickness values were computed using a uniform $72 \times 72$ mesh of MITC4S elements.

The results of Figure 17 show that also here the vibration mode oscillates more and more rapidly as the thickness becomes smaller. Moreover, the displacement amplitudes show a strong decay from the peak values which occur near the clamped edges. Observe that the range of these "edge effects" becomes approximatively halved when the thickness is reduced by a factor of ten. This indicates that the wavelength of the oscillations is proportional to $(L R t)^{1 / 3}$, i.e. to the characteristic length scale of hyperbolic shell layers [15].

The actual benchmark test was carried out again in two phases. In the first phase, the convergence of the lowest eigenvalue was assessed at $R / t=$ 100 by using a uniform $N \times N$ mesh sequence with $N=7,14,28$. Figure 18 is in line with the previous observations: the relative error of MITC4S is roughly four times smaller than the corresponding errors of the geometrically incompatible formulations.

In the second phase of the test, the asymptotic convergence was studied by employing $29 \times 29$ meshes which were refined uniformly towards the clamped edges. The refinement was done again so that the length of the element edges progress geometrically, the ratio of the largest and the smallest element side lengths being $r=3,6$, and 12 when $R / t=1000,10000$ and 100000 , respectively.

The lowest eigenvalues are reported in Table 6 . These results show that the accuracy of all geometrically incompatible formulations deteriorates on the anisotropically refined meshes as the parameter $t / R$ tends to the limit value of zero. MITC4S seems to be more or less uniformly convergent also here. 

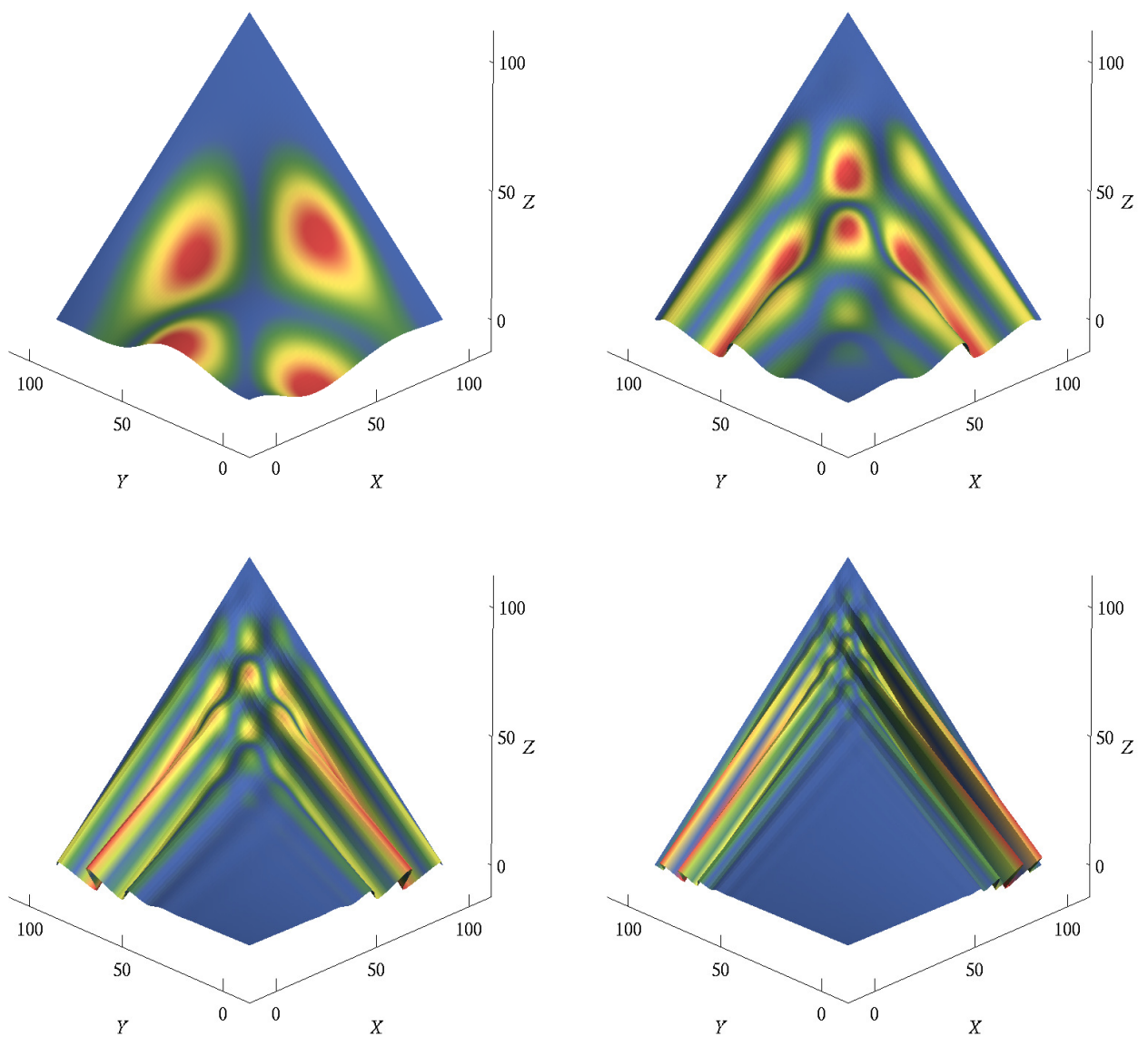

Figure 17: Lowest eigenmode of the hyperbolic paraboloid at $R / t=100$, 1000, 10000 and 100000: uniform $72 \times 72$ mesh of MITC4S elements.

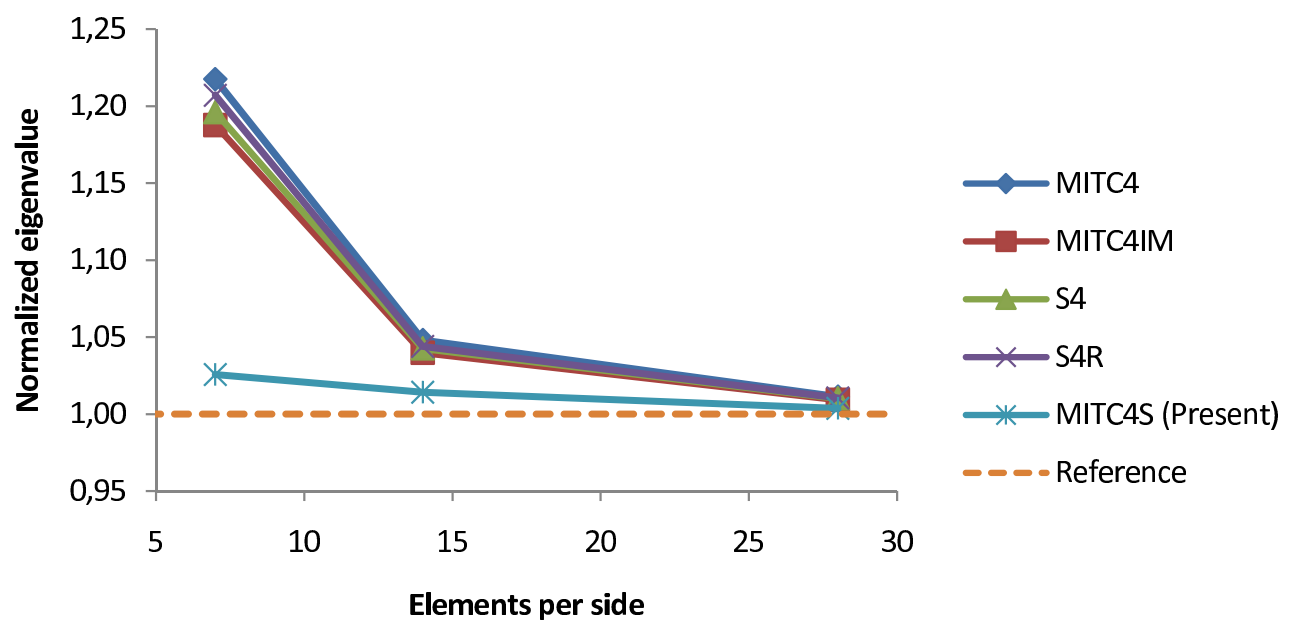

Figure 18: Convergence of the lowest eigenvalue of the hyperbolic paraboloid at $R / t=100$ : uniform $N \times N$ meshes with $N=7,14,28$. 


\begin{tabular}{llllll}
\hline$R / t$ & MITC4 & MITC4IM & S4 & S4R & MITC4S (Present) \\
\hline 1000 & 1.05 & 1.04 & 1.04 & 1.04 & 1.01 \\
10000 & 1.13 & 1.08 & 1.09 & 1.08 & 1.03 \\
100000 & 1.38 & 1.18 & 1.25 & 1.17 & 1.04 \\
\hline
\end{tabular}

Table 6: Lowest eigenvalues of the hyperbolic paraboloid (normalized to unity).

\section{Conclusions}

We have presented a four-node shell element (MITC4S) based on a refined shallow shell model to address the locking problems that arise in the approximation of shell layer or vibration modes using bilinear degenerated elements. Our approach was based on numerical experiments with two types of shell structures featuring the characteristic layer and vibration modes of parabolic and hyperbolic shells.

Concerning parabolic or cylindrical shells, the experiments show that

1. When approximating the characteristic layer or vibration mode of cylindrical shells, the basic bilinear degenerated formulation (MITC4) exhibits approximation failure, or locking, as the thickness tends to a limiting value of zero. In particular, this occurs if the mesh is refined anisotropically in accordance with the asymptotic nature of the layer/vibration mode.

2. The locking can be removed, at least on rectangular meshes, by employing the method of incompatible displacement modes (MITC4IM, $\mathrm{S} 4$ ), one point quadrature plus stabilization (S4R) or by reducing the geometrically compatible membrane strains (MITC4S).

The situation with hyperbolic shells is not so favorable because

1. None of the four-node elements in ABAQUS or ADINA appears to be asymptotically convergent, even for rectangular element shapes, when approximating the characteristic layer or vibration mode of hyperbolic shells on anisotropically refined meshes.

2. The method of incompatible displacement modes and the one point quadrature are able to alleviate the locking effect to some extent but MITC4S is more robust in this case.

Moreover, the convergence constant of MITC4S seems to be smaller than those of the non-curved elements. The present experiments indicate that in some cases the error in strain energy or eigenvalues may decrease even to one fourth if the effects of membrane and bending strain are coupled properly in the strain energy functional via geometric curvature. This means that the errors of stresses and vibration frequencies are effectively halved. 


\section{Appendix}

To solve the problems involving the circular cylindrical shell we introduce the distance measuring longitudinal and circumferential coordinates $X$ and $\varphi$. The equilibrium law of the shell deformation can be expressed in terms of the normal displacement $W$ and the normal force $Q$ by the so called DonnellVlasov equation

$$
\frac{E t^{3}}{12\left(1-\nu^{2}\right)} \Delta^{4} W+\frac{E t}{R^{2}} \frac{\partial^{4} W}{\partial X^{4}}=\Delta^{2} Q
$$

where $\Delta=\frac{\partial^{2}}{\partial X^{2}}+\frac{\partial^{2}}{\partial \varphi^{2}}$. The above equation is obtained from the exact but complex set of equations for cylindrical shells by neglecting some smalllooking terms, see e.g. [25] and the references therein. As emphasized in [26], (14) is valid if the circumferential wavelength of the deformation pattern is small compared to the radius $R$.

In our case the normal load can be expressed in the double Fourier series form $^{1}$

$$
Q(X, \varphi)=\sum_{m=1}^{\infty} \sum_{n=0}^{\infty}{ }^{\prime} Q_{m n} \cos \frac{\left(m-\frac{1}{2}\right) \pi X}{L} \cos \frac{2 n \varphi}{R}
$$

where the coefficients $Q_{m n}$ are defined as

$$
Q_{m n}=\frac{F}{\pi L R}
$$

corresponding to point loads of magnitude $F$ at $(0, n \pi R)$. Likewise, writing

$$
W(X, \varphi)=\sum_{m=1}^{\infty} \sum_{n=0}^{\infty}{ }^{\prime} W_{m n} \cos \frac{\left(m-\frac{1}{2}\right) \pi X}{L} \cos \frac{2 n \varphi}{R}
$$

we find from (14) that $W_{m n}$ takes the form

$$
W_{m n}=\frac{12\left(1-\nu^{2}\right) R^{2} Q_{m n}}{E t} \frac{\left(M^{2}+N^{2}\right)^{2}}{R^{2} t^{2}\left(M^{2}+N^{2}\right)^{4}+12\left(1-\nu^{2}\right) M^{4}}
$$

where $M=\left(m-\frac{1}{2} \pi\right) / L$ and $N=2 n / R$.

The solution to the complete set of cylindrical shell equations proceeds along the same lines. The longitudinal and circumferential displacement components $U$ and $V$ are represented as

$$
\begin{aligned}
& U(X, \varphi)=\sum_{m=1}^{\infty} \sum_{n=0}^{\infty}{ }^{\prime} U_{m n} \sin \frac{\left(m-\frac{1}{2}\right) \pi X}{L} \cos \frac{2 n \varphi}{R} \\
& V(X, \varphi)=\sum_{m=1}^{\infty} \sum_{n=1}^{\infty} V_{m n} \cos \frac{\left(m-\frac{1}{2}\right) \pi X}{L} \sin \frac{2 n \varphi}{R}
\end{aligned}
$$

\footnotetext{
${ }^{1}$ The notation $\sum_{n=0}^{\infty}$ ' indicates that the first term with $n=0$ is multiplied by one half.
} 


\begin{tabular}{lll}
\hline$R / t$ & Donnell-Vlasov & Novozhilov \\
\hline 100 & $-5.380 \times 10^{-6}$ & $-5.484 \times 10^{-6}$ \\
1000 & $-9.659 \times 10^{-4}$ & $-9.715 \times 10^{-4}$ \\
10000 & $-1.722 \times 10^{-1}$ & $-1.725 \times 10^{-1}$ \\
100000 & $-3.064 \times 10^{1}$ & $-3.066 \times 10^{1}$ \\
\hline
\end{tabular}

Table 7: Displacement of the cylinder under the point load.

and the unknown coefficients $U_{m n}, V_{m n}, W_{m n}$ can be found by solving a $3 \times 3$ matrix equation.

The displacement values under the point load are reported in Table 7 . These have been obtained by using the parameter values given in Section 3 and truncating the series (15) at $m=n=10000$. Two results are given here, one from the simplified Donnell-Vlasov equation (14) and the second from the 'exact' shell equations as represented by Novozhilov in [26]. We observe a small difference between the models which diminishes as $t$ decreases. The values of the Novozhilov model were anyway used as a reference in Section 3.

Equation (14) can be applied to the vibration problem as well if the normal surface load $Q$ is replaced by the radial inertia force

$$
Q=\rho t \frac{\partial^{2} W}{\partial \tau^{2}}
$$

and circumferential and longitudinal inertia forces are neglected. When $W$ is proportional to $\cos (\omega \tau)$, we obtain the equation

$$
\frac{E t^{3}}{12\left(1-\nu^{2}\right)} \Delta^{4} W+\frac{E t}{R^{2}} \frac{\partial^{4} W}{\partial X^{4}}=\omega^{2} \rho t \Delta^{2} W
$$

from which we find that the vibration frequencies of the principal modes in the expression (15) are given by

$$
\omega^{2}=\frac{E}{12\left(1-\nu^{2}\right) \rho R^{2}} \frac{R^{2} t^{2}\left(M^{2}+N^{2}\right)^{4}+12\left(1-\nu^{2}\right) M^{4}}{\left(M^{2}+N^{2}\right)^{2}}
$$

An examination of this expression reveals that $\omega^{2}$ attains its minimum at

$$
M \sim L^{-1}, \quad N \sim\left(L^{2} R t\right)^{-1 / 4}
$$

and that the minimum eigenvalue is of order

$$
\omega^{2} \sim \frac{E}{\rho} L^{-2} R^{-1} t
$$

If the tangential inertia forces are taken into account and the complete set of shell equations are employed, the vibration frequencies of the principal modes defined by (15) and (17) can be found by computing the eigenvalues 


\begin{tabular}{lll}
\hline$R / t$ & Donnell-Vlasov & Novozhilov \\
\hline 100 & $1.767 \times 10^{2}$ & $1.644 \times 10^{2}$ \\
1000 & $1.541 \times 10^{1}$ & $1.507 \times 10^{1}$ \\
10000 & $1.523 \times 10^{0}$ & $1.512 \times 10^{0}$ \\
100000 & $1.493 \times 10^{-1}$ & $1.490 \times 10^{-1}$ \\
\hline
\end{tabular}

Table 8: Lowest eigenvalues of the cylindrical shell problem.

\begin{tabular}{ll}
\hline$R / t$ & S8R 5 \\
\hline 100 & $-2.449 \cdot 10^{-6}$ \\
1000 & $-2.991 \cdot 10^{-4}$ \\
10000 & $-3.649 \cdot 10^{-2}$ \\
100000 & $-4.319 \cdot 10^{0}$ \\
\hline
\end{tabular}

Table 9: Displacement of the hyperbolic paraboloid under the point load.

of a $3 \times 3$ matrix. The two results whose difference vanishes as $t$ decreases, are shown in Table 8 . The values of the Novozhilov model were again used as a reference in Section 3.

The reference solutions for the two problems involving the hyperbolic paraboloid structure were computed numerically using very fine meshes of quadratic thin shell elements of ABAQUS. For the load problem, a $256 \times 256$ mesh was employed where all edges were refined as described in Section 3. The results are shown in Table 9.

For the vibration problem, respectively, we employed a uniform $256 \times 256$ mesh. The lowest eigenvalues are reported in Table 10.

\section{Acknowledgements}

The computations were carried out using ABAQUS Research Edition and 900 nodes version of ADINA System. The former was provided by CSC the Finnish IT center for science, whereas the latter was obtained directly from ADINA R \& D, Inc. The simulations were run mainly on a laptop computer, but the numerical reference solutions to the hyperbolic paraboloid test problems were computed using a HP CP4000 BL ProLiant supercluster available at CSC.

\begin{tabular}{ll}
\hline$R / t$ & $\mathrm{~S} 8 \mathrm{R} 5$ \\
\hline 100 & $1.306 \cdot 10^{3}$ \\
1000 & $2.203 \cdot 10^{2}$ \\
10000 & $3.539 \cdot 10^{1}$ \\
100000 & $6.207 \cdot 10^{0}$ \\
\hline
\end{tabular}

Table 10: Lowest eigenvalues of the hyperbolic paraboloid problem. 


\section{References}

[1] Lindberg G, Ohlson M, Cowper G. New developments in the finite element analysis of shells. National Research Council of Canada, Quarterly Bulletin of the Division of Mechanical Engineering and National Aeronautical Establishment 1969; 4:1-38.

[2] Cowper G, Lindberg G, Ohlson M. A shallow shell finite element of triangular shape. Int. J. Solids Structures 1970; 6:1133-1156.

[3] Ahmad S, Irons BM, Zienkiewicz OC. Analysis of thick and thin shell structures by curved finite elements. Int. J. Numer. Meth. Engng 1970; 2:419-451.

[4] MacNeal RH. A simple quadrilateral shell element. Comput. Struct. 1978; 8:175-183.

[5] Dvorkin EN, Bathe KJ. A continuum mechanics based four-node shell element for general non-linear analysis. Eng. Comput. 1984; 1:77-88.

[6] Niemi AH. Approximation of shell layers using bilinear elements on anisotropically refined rectangular meshes. Comput. Methods Appl. Mech. Engrg. 2008; 197:3964-3975.

[7] Simo JC, Fox DD, Rifai MS. On a stress resultant geometrically exact shell model. II. The linear theory; computational aspects. Comput. Methods Appl. Mech. Engrg. 1989; 73:53-92.

[8] Andelfinger U, Ramm E. EAS-elements for two-dimensional, threedimensional, plate and shell structures and their equivalence to HRelements. Int. J. Numer. Meth. Engng 1993; 36:1311-1337.

[9] Bathe KJ. Finite Element Procedures. Prentice Hall: Englewood Cliffs, 1996.

[10] Gebhardt H, Schweizerhof K. Interpolation of curved shell geometries by low order finite elements - errors and modifications. Int. J. Numer. Meth. Engng 1993; 36:287-302.

[11] Malinen M. On the classical shell model underlying bilinear degenerated shell finite elements: general shell geometry. Int. J. Numer. Meth. Engng 2002; 55:629-652.

[12] Hughes TJR. The Finite Element Method. Linear Static and Dynamic Finite Element Analysis. Prentice-Hall: Englewood Cliffs, New Jersey, 1987.

[13] Zienkiewicz O, Taylor R. The finite element method. Volume 2: Solid mechanics. Fifth edition. Butterworth-Heinemann: Oxford, 2000. 
[14] MacNeal RH. Finite elements: their design and performance. Marcel Dekker, Inc.: New York, 1994.

[15] Pitkäranta J, Matache AM, Schwab C. Fourier mode analysis of layers in shallow shell deformations. Comput. Methods Appl. Mech. Engrg. 2001; 190:2943-2975.

[16] Niemi AH, Pitkäranta J. Bilinear finite element for shells: isoparametric quadrilaterals. Int. J. Numer. Meth. Engng 2008; 75:212-240.

[17] Belytschko T, Lin JI, Tsay CS. Explicit algorithms for the nonlinear dynamics of shells. Comput. Methods Appl. Mech. Engrg. 1984; 42:225251.

[18] Lyly M, Stenberg R, Vihinen T. A stable bilinear element for the reissner-mindlin plate model. Comput. Methods Appl. Mech. Engrg. 1993; 110:343-357.

[19] ADINA R \& D, Inc.. ADINA Theory and Modeling Guide: ARD 08-7 February 2008.

[20] Dassault Systèmes. ABAQUS Theory Manual (v6.7) 2007.

[21] Niemi AH, Pitkäranta J, Hakula H. Benchmark computations on pointloaded shallow shells: Fourier vs. FEM. Comput. Methods Appl. Mech. Engrg. 2007; 196:894-907.

[22] Briassoulis D. Testing the asymptotic behaviour of shell elementsPart 1:the classical benchmark tests. Int. J. Numer. Meth. Engng 2002; 54:421-452.

[23] Niemi AH, Pitkäranta J, Hakula H. Point load on a shell. Numerical mathematics and advanced applications. Springer: Berlin, 2008; 819826.

[24] L Beirão da Veiga, Hakula H, Pitkäranta J. Asymptotic and numerical analysis of the eigenvalue problem for a clamped cylindrical shell. I.M.A.T.I.-C.N.R. 2007; :1-17.

[25] Lukasiewicz S. Local loads in plates and shells. Sijthoff \& Noordhoff: The Netherlands, 1979.

[26] Novozhilov VV. The Theory of Thin Shells. P. Noordhoff, Ltd. Groningen: The Netherlands, 1959. 
(continued from the back cover)

A555 Juho Könnö, Rolf Stenberg

Finite Element Analysis of Composite Plates with an Application to the Paper Cockling Problem

December 2008

A554 Lasse Leskelä

Stochastic relations of random variables and processes

October 2008

A553 Rolf Stenberg

A nonstandard mixed finite element family

September 2008

A552 Janos Karatson, Sergey Korotov

A discrete maximum principle in Hilbert space with applications to nonlinear cooperative elliptic systems

August 2008

A551 István Faragó, Janos Karatson, Sergey Korotov

Discrete maximum principles for the FEM solution of some nonlinear parabolic problems

August 2008

A550 István Faragó, Róbert Horváth, Sergey Korotov Discrete maximum principles for FE solutions of nonstationary diffusion-reaction problems with mixed boundary conditions

August 2008

A549 Antti Hannukainen, Sergey Korotov, Tomás Vejchodský

On weakening conditions for discrete maximum principles for linear finite element schemes

August 2008

A548 Kalle Mikkola

Weakly coprime factorization, continuous-time systems, and strong- $H^{p}$ and Nevanlinna fractions

August 2008

A547 Wolfgang Desch, Stig-Olof Londen

A generalization of an inequality by N. V. Krylov

June 2008 


\section{HELSINKI UNIVERSITY OF TECHNOLOGY INSTITUTE OF MATHEMATICS RESEARCH REPORTS}

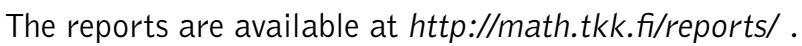

The list of reports is continued inside the back cover.

A561 Antti Hannukainen, Sergey Korotov, Michal Krizek

On nodal superconvergence in 3D by averaging piecewise linear, bilinear, and trilinear FE approximations

December 2008

A560 Sampsa Pursiainen

Computational methods in electromagnetic biomedical inverse problems

November 2008

A559 Sergey Korotov, Michal Krizek, Jakub Solc

On a Discrete Maximum Principle for Linear FE Solutions of Elliptic Problems with a Nondiagonal Coefficient Matrix

November 2008

A558 José Igor Morlanes, Antti Rasila, Tommi Sottinen

Empirical evidence on arbitrage by changing the stock exchange

December 2008

A556 Lourenço Beirão da Veiga, Jarkko Niiranen, Rolf Stenberg

A posteriori error analysis for the Morley plate element with general boundary conditions

December 2008

ISBN 978-951-22-9719-1 (print)

ISBN 978-951-22-9720-7 (PDF)

ISSN 0784-3143 (print)

ISSN 1797-5867 (PDF) 